\title{
RFID unreliable data filtering by integrating adaptive sliding window and Euclidean distance
}

\author{
Li-Lan Liu $\cdot$ Zi-Long Yuan $\cdot$ Xue-Wei Liu • \\ Cheng Chen $\cdot$ Ke-Sheng Wang
}

Received: 28 January 2014/ Accepted: 14 May 2014/Published online: 5 June 2014

(C) Shanghai University and Springer-Verlag Berlin Heidelberg 2014

\begin{abstract}
Through improving the redundant data filtering of unreliable data filter for radio frequency identification (RFID) with sliding-window, a data filter which integrates self-adaptive sliding-window and Euclidean distance is proposed. The input data required being filtered have been shunt by considering a large number of redundant data existing in the unreliable data for RFID and the redundant data in RFID are the main filtering object with utilizing the filter based on Euclidean distance. The comparison between the results from the method proposed in this paper and previous research shows that it can improve the accuracy of the RFID for unreliable data filtering and largely reduce the redundant reading rate.
\end{abstract}

Keywords Radio frequency identification (RFID) .

Adaptive sliding window $\cdot$ Euclidean distance .

Redundant data

\section{Introduction}

Radio frequency identification (RFID) is a non-contact automatic identification technology which is able to automatically identify the target objects by radio frequency signal,quickly carry goods monitoring, positioning, tracking and exchanging data, process without manual intervention

L.-L. Liu $(\bowtie) \cdot$ Z.-L. Yuan · X.-W. Liu · C. Chen

Shanghai Key Laboratory of Intelligent Manufacturing and Robotics, Shanghai University, Shanghai 200072, People's Republic of China

e-mail: lancy@shu.edu.cn

K.-S. Wang

Department of Production and Quality Engineering, Norwegian University of Science and Technology, Trondheim, Norway and work in the harsh environment. A complete RFID system contains three parts: reader, electronic tag, and antenna. The reader transmits radio frequency signal by antenna, and the electronic tag placed in the tracked object induces current after receiving signal to return a response signal to the reader, then this system realizes the object's tracking, detection, identification, data acquisition and control by decoding response signal [1,2].

With the development of internet technology, RFID technology has been widely used in the fields of supply chain logistics of manufacturing, manufacturing and traffic management. However, due to the difference of tag conditions and physical characteristics of RF signal, the raw data collected by reader are unreliable, including false positive, false negative and false redundancies. The unreliability of data directly limits the promotion and application of RFID technology. Therefore, it is very important to filter the raw data in RFID application.

Currently, data filtering technology in the application of RFID has made some progress. The optimization method presented in Ref. [3], with a record of repeated optimization queue instead of a fixed size window, shows that the larger priority queue is, the higher the detection accuracy of the algorithm is, but it takes longer running time. Jeffery et al. [4] proposed an adaptive unreliable RFID data flow smooth method, which is mainly for a single reader, and they just proposed to filter data of multireaders. Chen et al. [5] used the method of Bayesian inference based on data cleaning on existing data cleaning technology which could not accurately restore data location information and at the same time provide position information to filter the data effectively. However this method is heavily dependent on specific reader deployment topology. Carbunar et al. [6] discussed the cleaning problem of the redundant data by detecting and silencing 


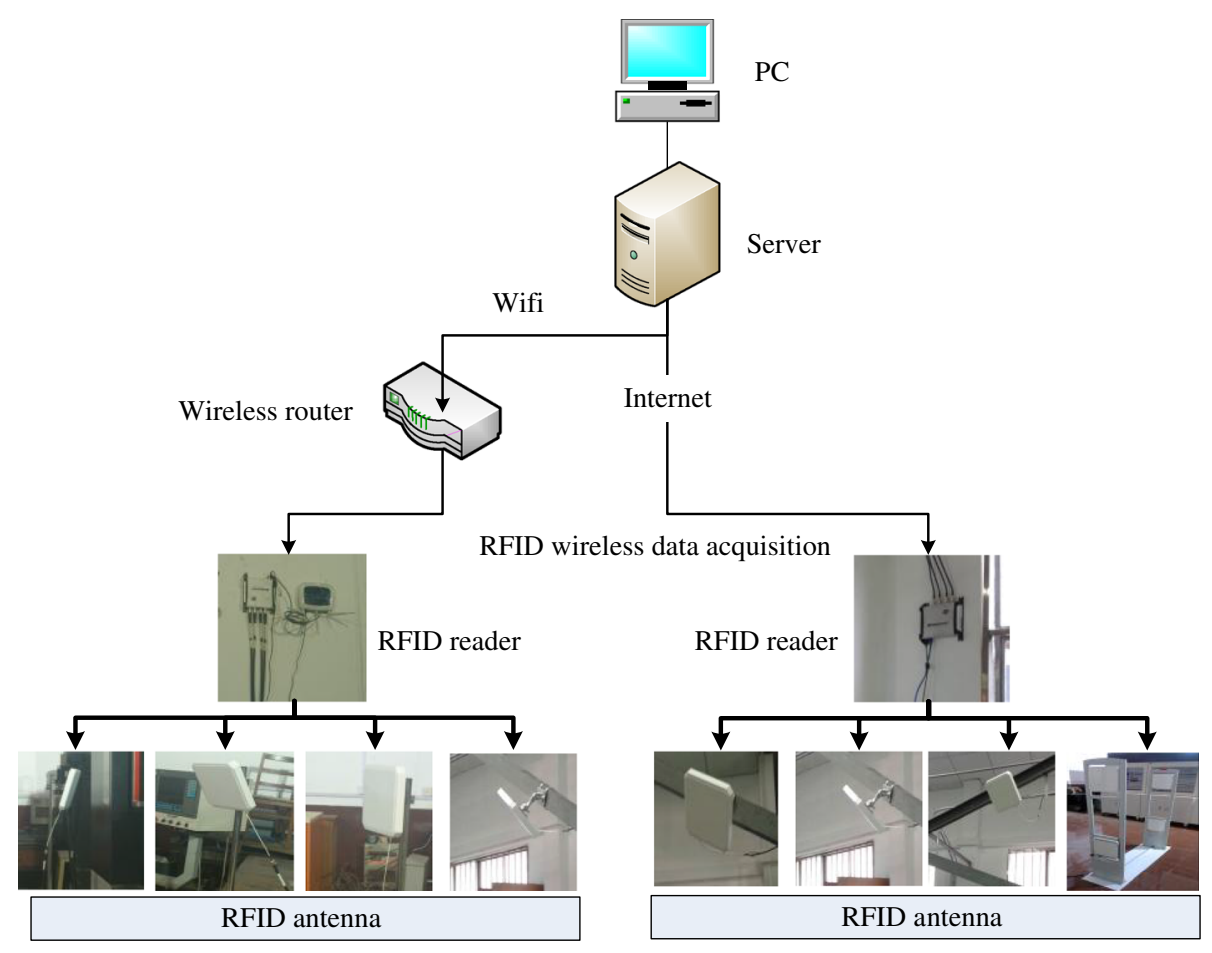

Fig. 1 RFID system hardware architecture diagram

the redundant reader, but the redundant reader elimination (RRE) algorithm proposed in this literature could not solve practical problems that multiple adjacent readers had to work simultaneously.

In accordance with the above case, this paper has proposed a new method for RFID unreliable data filtering, which is based on adaptive cleaning algorithm SMURF (statistical smoothing for unreliable RFID data) for RFID data and combines the advantages of adaptive sliding window and Euclidean distance. The method fully considering the RFID unreliable data in the system with a large number of redundant data problems is supposed to shunt the filtered data. In addition, it introduces redundant data filtering method based on Euclidean distance and focuses on RFID redundant data filtering. Finally, comparative analysis of the experimental data shows that the method can improve the accuracy of the RFID unreliable data filtering and largely reduce the percentage of false redundancies while maintaining the time-consuming data filtering.

\section{RFID real-time data acquisition}

Any RFID data acquisition system is composed of three major assemblies, i.e., RFID tags, RFID readers and computer control system. RFID tags are attached in logistics monitoring objects of the movement, and the reader is generally fixed at a fixed location or movable location according to the special function of the definition, called as the data collection points. During the data collection process, the data are transmitted to the computer. Then the computer extracts station number and process condition through the reader number. At the same time, the material characteristics information and material status flag information are extracted from the tag data, and written to real-time database together with the data acquisition time. Finally the status information is written to corresponding tag or database table to complete the acquisition of a data item. The hardware frame of real-time data acquisition system is shown in Fig. 1.

\section{RFID data filtering model}

3.1 RFID unreliable data filtering based on the adaptive sliding window algorithm

When using the fixed sliding window cleaning method to filter the RFID data streams, the window size is difficult to be decided. This problem was solved [7] by an adaptive smoothing filter for RFID data cleaning-SMURF. The method utilized sample to seek the suitable time window size for the application system. Unlike the traditional data filtering technology, this model does not apply smooth window parameters in data application system, but changes the window size automatically and constantly to adapt with the change of the system cycle of the observed data $[8,9]$. 
SMURF mainly contains two filtering mechanisms: single tag reading filtering mechanism and multi tags aggregation filtering mechanism [10].

\subsubsection{Single tag reading filtering}

Bernoulli binomial model is used to deal with a single tag in the literature. When at least one tag is read in a sliding window, we define the window size as $w_{i}$ and average reading rate in each reading period window as $p_{i}^{\text {avg }}$, and set that SMURF using statistic mechanism based on random sampling. Each cycle, SMURF regards $t_{i}$ as independent Bernoulli sampling times and $s_{i}$ as reading times, so $p_{i}=\frac{s_{i}}{t_{i}}$. Then in the whole window $w_{i},\left|s_{i}\right|$ means the number of times of a tag appears in the window, and its average reading rate is defined as

$p_{i}^{\text {avg }}=\frac{\sum_{t=1}^{w} p_{i, k}}{\left|s_{i}\right|}$.

To ensure the integrity of reading data, according to the Bernoulli probability model, the window size is

$w_{i} \geq\left(\frac{\ln (1 / \delta)}{p_{i}^{\text {avg }}}\right)$,

where $\delta$ represents a negative reading rate in the filtered data.

With the integrity of tag data solved, according to the central limit theorem, the condition for tag changing is

||$s_{i}\left|-w_{i} p_{i}^{\text {avg }}\right|>2 \sqrt{w_{i} p_{i}^{\text {avg }}\left(1-p_{i}^{\text {avg }}\right)}$.

\subsubsection{Multi tags aggregation filtering}

The model of $\pi$-estimator is used in the literature to handle multiple tags gathering questions to accurately count the number of tags in the window. The window size is $w_{i}$, and the reading rate of tags in window is $p_{i}^{\text {avg }}$. Then the probability that the tags appear at least once in the window is given as

$\pi_{i}=1-\left(1-p_{i}^{\text {avg }}\right)^{w}$

The total number of tags in the window is

$N_{\mathrm{w}}=\sum_{i \in S_{\mathrm{w}}} \frac{1}{\pi_{i}}$.

Definition

$\operatorname{var}\left(N_{\mathrm{w}}\right)=\sum_{i \in S_{\mathrm{w}}} \frac{1-\pi_{i}}{\pi_{i}^{2}}$

where $S_{\mathrm{w}}$ is the tag set detected by the window. The adjustment of window size for multi tags aggregation filtering is the same as single ones, and the condition for tag dynamic change is given as

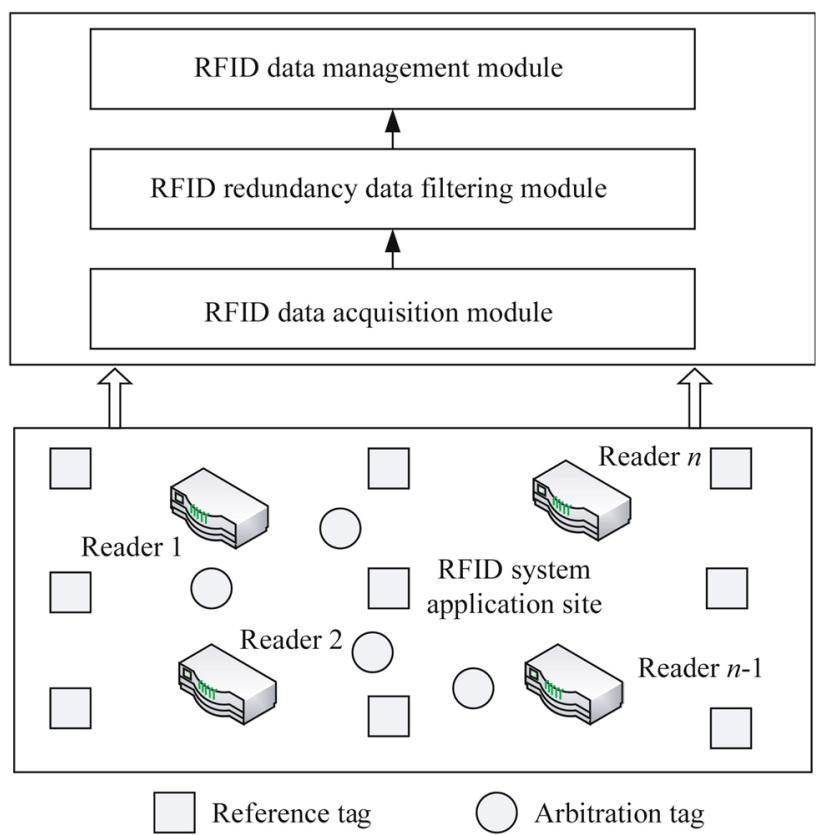

Fig. 2 RFID redundant data filtering and reference tag deployment diagram

$\left|N_{\mathrm{w}}-N_{\mathrm{w}^{\prime}}\right|>2\left(\sqrt{\operatorname{var}\left(N_{\mathrm{w}}\right)-\operatorname{var}\left(N_{\mathrm{w}^{\prime}}\right)}\right)$,

where $N_{\mathrm{w}^{\prime}}$ is the number of tags in the first $1 / 2$ window.

\subsection{RFID redundant data filtering based on Euclidean distance}

For any two tuples, $<\mathrm{TID}_{i}, \mathrm{RID}_{i}$, Time $_{i}>$ and $<\mathrm{TID}_{j}$, $\mathrm{RID}_{j}, \mathrm{Time}_{j}>$, which come from the same cross reader space group, if $\left(\mathrm{TID}_{i}=\mathrm{TID}_{j}\right) \wedge\left(\mathrm{RID}_{i} \neq \mathrm{RID}_{j}\right)$ and time constraint $\left(\right.$ Time $_{i}=$ Time $\left._{j}\right) \vee\left(\mid\right.$ Time $_{i}-$ Time $_{j} \mid \leq$ Threshold $)$ are satisfied, the data collected via multiple readers will be redundant. Therein, TID means tag identifier, which represents a unique identifier of the identified object; RID presents the reader's unique identifier; threshold is the time threshold. The method of judging the vesting reader based on Euclidean distance is shown as follows [11-14].

(i) Extend tuple data

Extend the original data tuple by introducing RSSI (receiving information strength) properties. Derivate the relative position of tag objects by using RSSI values of the location of reader and tag which is already known.

(ii) Calculate signal strength $U_{i j}$

RFID reader $j$ monitors the pending arbitration tag $i$. Then, the signal generates and its strength $U_{i j}$ in time sliding window $[t, t+w]$ is calculated. RSSI 


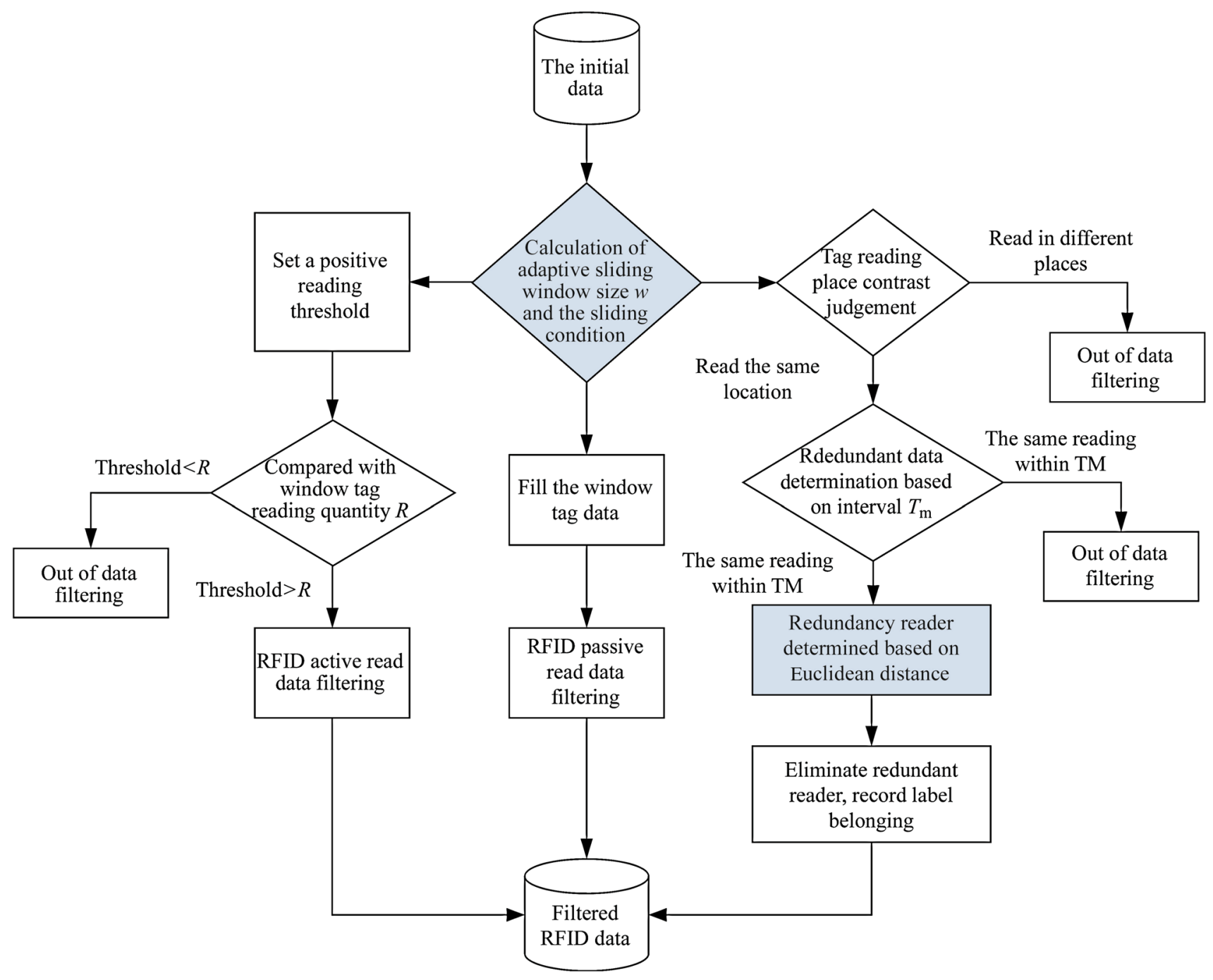

Fig. 3 HSMURFED method flowchart

value from several cross information tuples in the window is regarded as the sample space whose populations obey normal distribution. Then, the maximum likelihood estimation value of $\mu$ is calculated and defined as the signal intensity $U_{i j}$ of the tag in time sliding window [15].

(iii) Deploy reference tag

Referring tag is an additional fixed tag, whose signal strength is regarded as a reference signal strength point of the reader attribution, as shown in Fig. 2.

(iv) Calculate the signal strength vector

Calculate the signal strength vector $\boldsymbol{V}_{j}=$ $\left(V_{j 1}, V_{j 2}, \cdots, V_{j m}\right)$ of the $m$ reference tags in time sliding window $[t, t+w]$, which belongs to reader $j$.

(v) Calculate the Euclidean distance of signal strength vector
$E_{i j}=\sqrt{\sum_{k=1}^{M}\left(U_{i j}-V_{i k}\right)^{2}}, \quad i \in(1, S), \quad j \in(1, S)$.

Assume that there are $n$ readers, $n \times m$ fixed reference tags which the attributions are already known. $V_{i k}, j \in(1, n), k \in(1, m)$ is defined as signal strength of the reference $\operatorname{tag} k$ in the reader $j$.

\subsection{Synthesis method of SMURF and Euclidean distance for RFID unreliable data filtering}

SMURF cleaning method based on adaptive sliding window does not need application program to define the window size. Alternatively, the window size is calculated 
adaptively based on data quality in the window. In this way, the negative reading and positive reading caused by unreasonable window size of fixed-length sliding window size method can be improved. However, this method only makes an adaptive improvement for the size of the sliding window on the whole without improving the redundant read in filtering methods $[16,17]$. Therefore, there are some limitations in the application of this system. In this paper, on the basis of SMURF method, a RFID unreliable data filtering method is proposed combining the SMURF with Euclidean distance (HSMURFED).

Unreliable data filtering for RFID is divided into two subproblems in order to be solved separately. The process of RFID unreliable data filtering method (HSMURFED) based on an SMURF and Euclidean distance is shown in Fig. 3.

Step 1 Calculate the average reading rate of $\operatorname{tag} p_{i}^{\text {avg }}$.

(i) Define tag reading rate

Set $p_{i, k}$ is ratio of the successful information feedback number for tag $i$ in the reading cycle $k$ and the number of queries contained reading cycle.

$p_{i, k}=\frac{\text { count__eturn }_{k}}{\text { count_probe }}$,

where count_return ${ }_{k}$ is the feedback time of $\operatorname{tag} i$ in reading cycle $k$, and count_probe is the number of queries cycle contained in the read cycle. Hence, there is $0 \leq p_{i, k} \leq 1$.

(ii) Calculation of the average reading rate of tag $p_{i}^{\text {avg }}$

$p_{i}^{\text {avg }}$ is the ratio of the sum of reading rate to each reading cycle in the sliding window to the number of reading cycle that the window contains.

$p_{i}^{\mathrm{avg}}=\frac{\sum_{i=1}^{k=w} p_{i, k}}{w}$.

where $w$ is the number of read cycles contained in the window.

Step 2 Calculate the adaptive window size $w_{i}$ and condition of the sliding window according to Eqs. (1) and (2) as well as unreliable data type determination, and take the appropriate processing methods, such as the data quantity of the sliding window is less than threshold, then choose a positive reading data filtering method.

Step 3 RFID unreliable data filtering method, which is divided into three types: false negative data filtering, false positive data filtering and false redundancies data filtering. The explanation is as follows [18].

(i) False negative data filtering

The principle of false negative data filtering is at any time, the reading data of tag are considered existing in the whole window as long as one reading of its exists in the window. Then the window outputs this reading. With the window sliding, the negative reading data in the original data are filled up.

(ii) False positive data filtering

The principle of false positive data filtering is that the incidence of false positive data rate is lower than the correct one so a false positive threshold is set. If in the window reading data quantity is less than threshold, the read will be considered as false positive. When windows receive new reading $R$ each time, the whole window will be scanned and the quantity of the same reading as $R$ in the window will be calculated. If this number is greater than the threshold, mark $R$ as non-active read, then output it when $R$ is expired from the window.

(iii) False redundancies data filtering

Due to a higher percentage of redundant data in the system, the traditional method of setting time interval only filters the data redundancy, but cannot effectively filter the reader redundancy. Therefore, the Euclidean distance method is increased for redundancy filtering. Concrete steps are as follows [19].

(a) When the reader reads a tag, compared to it « a tag, compared to its last reading place, if different, jump out the decision, and if the same, come into the following steps.

(b) Set the time interval $T_{\mathrm{m}}$, if the read data are different in the $T_{\mathrm{m}}$, jump out; if the read data are the same, transfer to the following steps that can filter the redundant data in the data redundancy.

(c) Determine redundant data reader.

(d) Vest reader to determine: the introduction of the Euclidean distance determinates the attribution of reader.

Step 4 Complete RFID unreliable data filtering, and output the filtered data.

\section{Experimental application and comparative analysis}

\subsection{Experimental data}

This paper simulates a test: a tag on the two readers reads 1,000 query cycles. Because of the overlap among read ranges, this experiment can test the redundant data filtering effect. In the inquiry period, 0-5 tags are placed randomly. When the number of tags reader read is different from the 
Table 1 Tag analog state in reader1

\begin{tabular}{|c|c|c|}
\hline Inquiry cycle & Read range & Metal interference \\
\hline $1-25$ & $\checkmark$ & \\
\hline \multicolumn{3}{|l|}{$26-50$} \\
\hline $51-75$ & $\checkmark$ & \\
\hline \multicolumn{3}{|l|}{$76-100$} \\
\hline $101-125$ & $\boldsymbol{V}$ & \\
\hline \multicolumn{3}{|l|}{$126-150$} \\
\hline $151-175$ & $\checkmark$ & \\
\hline \multicolumn{3}{|l|}{ 176-200 } \\
\hline $201-225$ & $\checkmark$ & \\
\hline $226-250$ & $\checkmark$ & \\
\hline \multicolumn{3}{|l|}{$251-275$} \\
\hline $276-300$ & $\checkmark$ & \\
\hline \multicolumn{3}{|l|}{$301-325$} \\
\hline $326-350$ & $\checkmark$ & \\
\hline \multicolumn{3}{|l|}{$351-375$} \\
\hline $376-400$ & $\checkmark$ & \\
\hline \multicolumn{3}{|l|}{$401-425$} \\
\hline $426-450$ & $\checkmark$ & \\
\hline \multicolumn{3}{|l|}{$451-475$} \\
\hline $476-500$ & $\boldsymbol{V}$ & \\
\hline $501-525$ & & $\checkmark$ \\
\hline $526-550$ & $\boldsymbol{V}$ & \\
\hline \multicolumn{3}{|l|}{$551-575$} \\
\hline $576-600$ & $\checkmark$ & $\checkmark$ \\
\hline \multicolumn{3}{|l|}{$601-625$} \\
\hline $626-650$ & $\checkmark$ & $\checkmark$ \\
\hline $651-675$ & $\boldsymbol{v}$ & \\
\hline \multicolumn{3}{|l|}{$676-700$} \\
\hline $701-725$ & $\checkmark$ & $\checkmark$ \\
\hline $726-750$ & $\checkmark$ & \\
\hline \multicolumn{3}{|l|}{$751-775$} \\
\hline $776-800$ & $\checkmark$ & \\
\hline $801-825$ & $\checkmark$ & $\checkmark$ \\
\hline \multicolumn{3}{|l|}{$826-850$} \\
\hline $851-875$ & $\checkmark$ & $\checkmark$ \\
\hline $876-900$ & $\checkmark$ & \\
\hline \multicolumn{3}{|l|}{$901-925$} \\
\hline $926-950$ & $\checkmark$ & \\
\hline $951-975$ & $\checkmark$ & $\boldsymbol{V}$ \\
\hline 976-1,000 & & \\
\hline
\end{tabular}

actual number of tags, record the results. Besides, adding in some period covered metal interference, analog tag states are listed in Tables 1 and 2. The "201-440" cycles are used for simulating tag's static characteristics, keeping tags in the detection range of the reader. Considering the dynamic characteristic of the tag, the tag is simulated to move into and out of the read write device detection range
Table 2 Tag analog state in reader 2

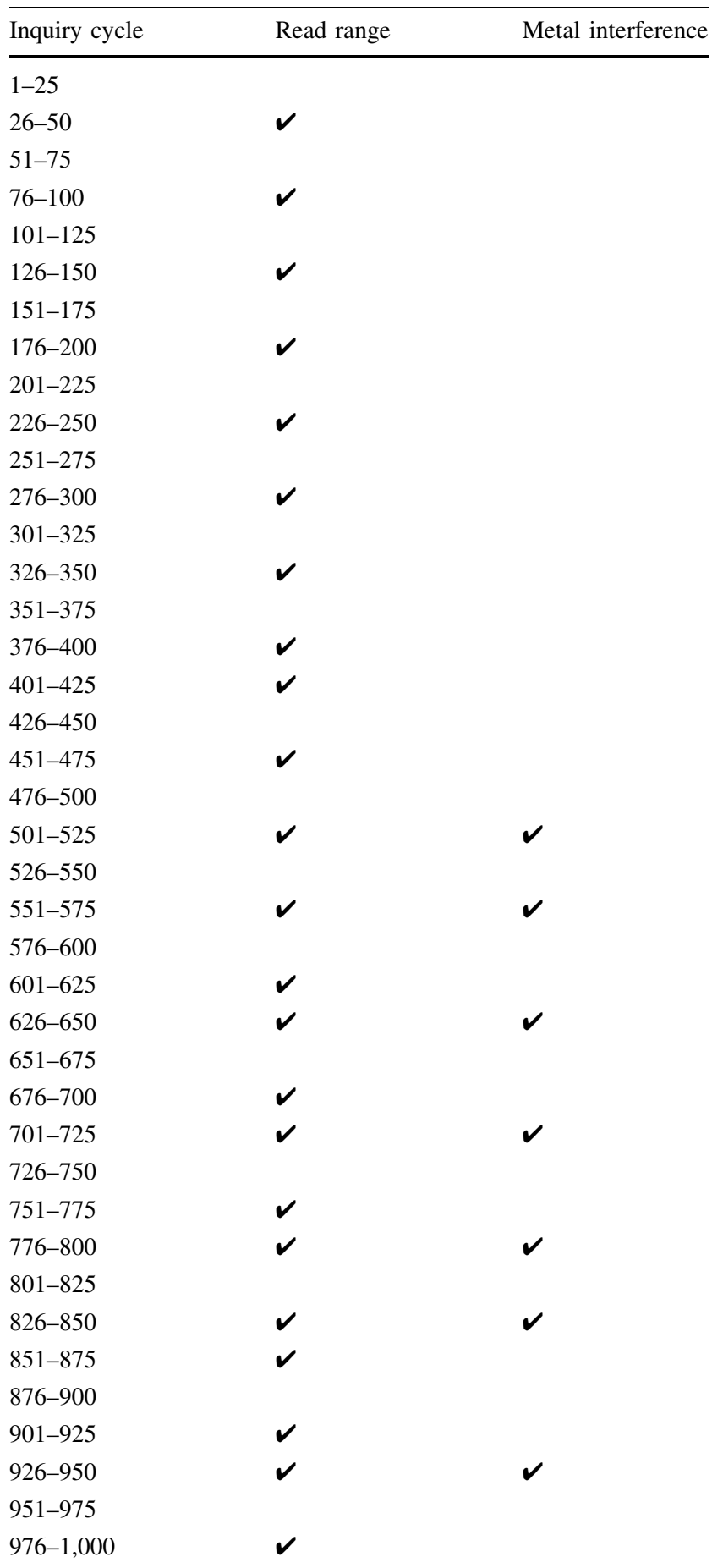

by certain rules. For example "1-25" in Table 1 query cycle appears in reader reading range, while the "26-50" query cycle tag does not appear in reader reading range. In addition to considering the tag's diversity of environment, The detected probability of the tag in some cycle is randomly reduced to simulate the metal cover after 500 cycles (e.g., "626-650" periods). 


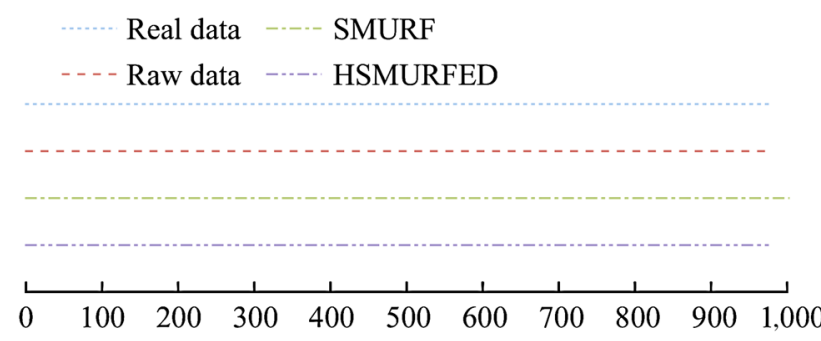

(a) Reader 1

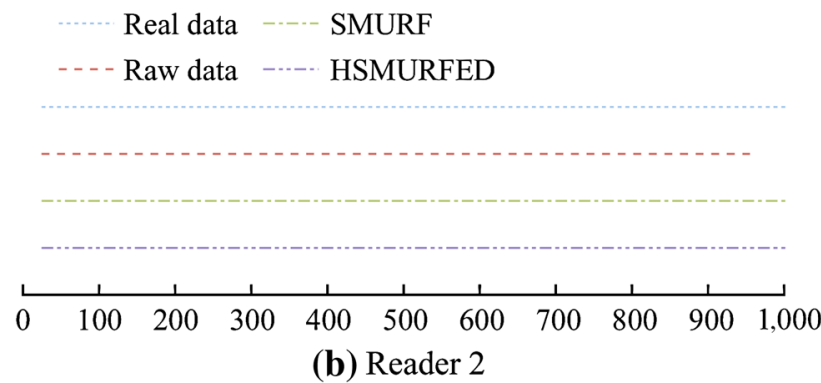

Fig. 4 Comparative data filtering result

In conclusion, the experimental data take not only static characteristics, but also dynamic characteristics of the tag into consideration, and these data also simulate redundant read and low detection rate case (such as metal cover) [20].

\subsection{Comparative analysis of the accuracy of data filtering}

The tag is filtered which comes in and out of reader's detection zone frequently during the 1,000 query cycles in Table 1 by using RFID data cleaning method based on adaptive window size (SMURF) and data filtering method based on HSMURFED respectively. Figure 4 shows the real data, raw data and filtering results of the above two methods for filtering raw data.

It is supposed that reading data results may be affected by the redundancy between two readers, and the SMURF method and HSMURFED methods may have a negative reading. However, Fig. 4 is not sufficient to explain the performance of each method of filtering results. The following discussion will continue to compare the various methods of data filtering effect.

To measure the filtering effect of RFID unreliable data on the accuracy of reading, the accuracy of the ratio that is the ratio of the number of filtered data equals to the real data to the total number of data, denoted as $A_{\text {cc }}$. With $N$ test data, the number of the filtered data equal to the real values is $D$, and the cleaning result accuracy is defined as $A_{\mathrm{cc}}=\frac{D}{N}$.

According to Fig. 5, the conclusions are as following:

(i) In 0-150 query cycles, data filtering accuracy rate in three cases is $100 \%$.

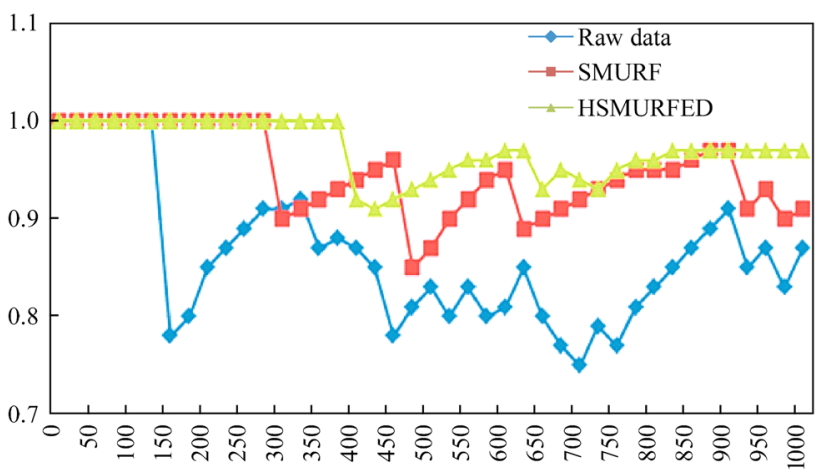

(a) Reader 1

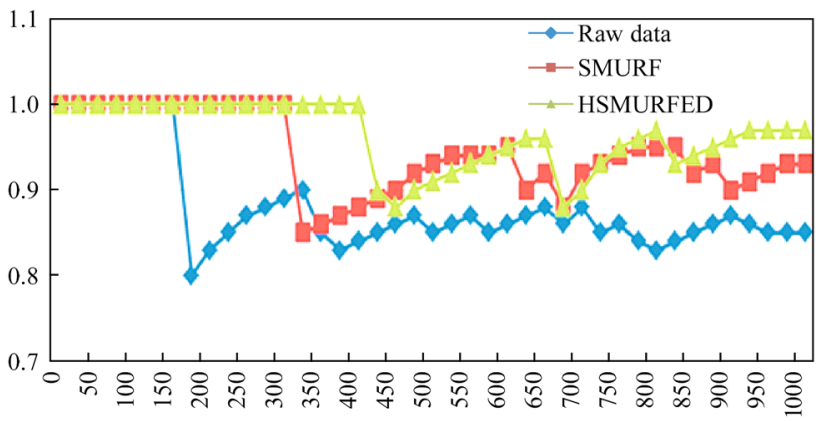

(b) Reader 2

Fig. 5 Data filtering accuracy rate comparison

(ii) In 151-175 query cycles, negative reading appears in original cases, and the accuracy rate decreases.

(iii) In 326-350 query cycles, tags are removed from the reading range, and SMURF method does not filter redundant data effectively, resulting in false positive in reader 1 , thus SMURF accuracy rate is from 1 down to 0.9 .

(iv) In 201-400 query cycles, the accuracy rate of SMURF methods has increased because the tag has been in the detection range of the reader.

(v) In 500-1,000 query cycles, due to the addition of the metal cover jamming, the accurate rates of the 3 methods are decreased. However the accuracy rates of SMURF method and HSMURFED method after a period of adaptation are on the rise.

After the 1,000 inquiry periods, the accuracy of three filtering methods is as follows:

(i) Reader 1

The final accuracy rates are respectively $0.87,0.91$ and 0.96 by using raw data, SMURF method and HSMURFED method. Average accuracy rates are respectively $0.861,0.935$ and 0.97 by using raw data, SMURF method and HSMURFED method.

(ii) Reader 2

The final accuracy rates are respectively $0.85,0.93$ and 0.97 by using raw data, SMURF method and HSMURFED method. Average accuracy rates are 


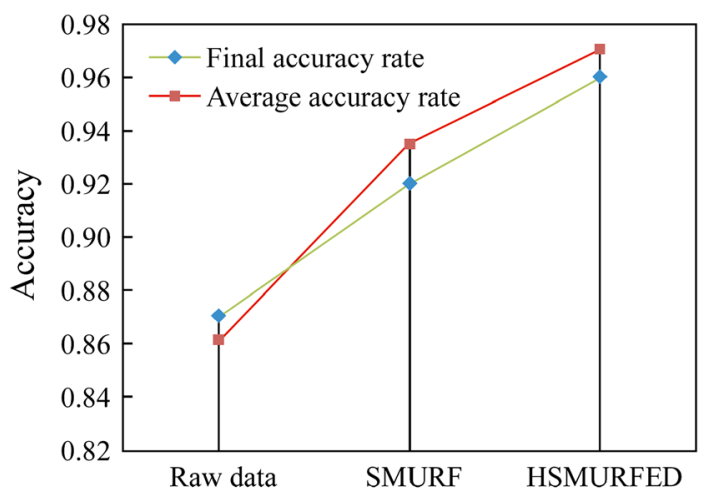

(a) Reader 1

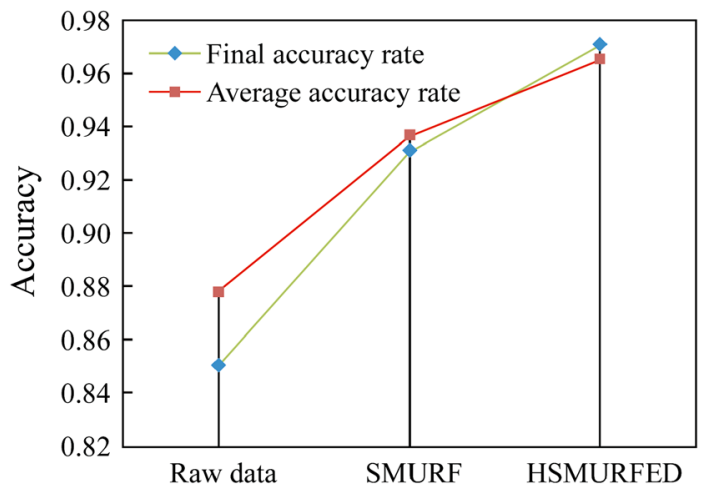

(b) Reader 2

Fig. 6 Three methods of filtering result

respectively $0.878,0.946$ and 0.965 by using raw data, SMURF method and HSMURFED method.

Comparison of three methods of filtering accuracy rates in the two readers is shown in Fig. 6.

According to Fig. 6, the conclusions are as follows:

(i) Both the final accuracy and average accuracy of HSMURFED method in the process of data filtering are better than the other two methods.

(ii) HSMURFED method only improves the redundancy data filtering on the basis of the SMURF method, so presumably HSMURFED method of filtering effect is caused by filtering the redundant data effectively.

In summary, according to HSMURFED data filtering method presented in this paper, the accuracy rates of dynamic tag and static tag after filtering are slightly higher than the other two methods.

\subsection{Comparative analysis of false redundancies}

To measure the cleaning method of false positive, false negative and false redundancies, the following three concepts are proposed: percentage of false negative,

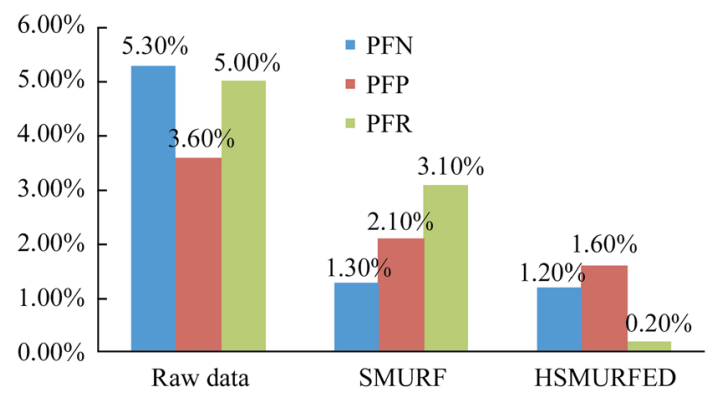

(a) Reader 1

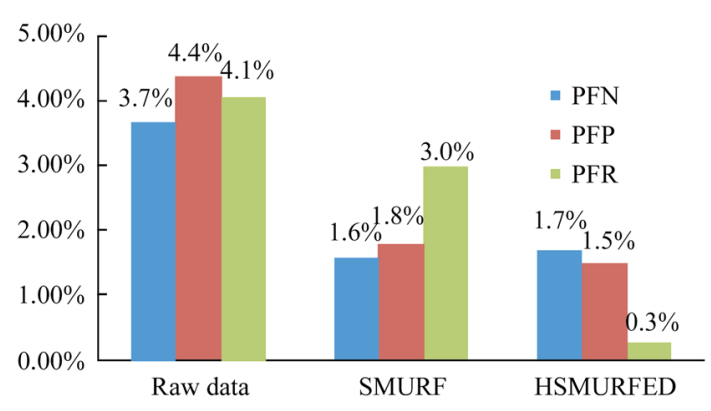

(b) Reader 2

Fig. 7 Percentage of three types of data

percentage of false positive and percentage of false redundancies, which can be defined as follows:

(i) Percentage of false negative

After filtration, the frequency of tag unread by the reader accounting for the total frequency of the filter when tags are within the scope of the reader is denoted as PFN.

(ii) Percentage of false positive

After filtration, the frequency of tag read by the reader accounting for the total frequency of the filter when tags are not within the scope of the reader is denoted as PFP.

(iii) Percentage of false redundancies

The frequency of one tag read by two readers simultaneously (reader redundancy) or one reader repeatedly (data redundancy) accounting for the total frequency of the filter is denoted as PFR.

According to Fig. 7, the conclusions are as follows:

(i) Percentage of false redundancies of HSMURFED filter method is the lowest comparing with raw data and SMURF filter method.

(ii) The filtering results of three kinds of unreliable data using the SMURF filtering method are improved, especially false negative and false positive. The SMURF method only deals with data redundancy, but does not take the reader redundancy into account. Thus the redundant data filtering effect is not ideal. 
(iii) Due to the introduction of Euclidean distance method, HSMURFED can deal with reader redundancy filtering, and sharply reduce the redundancy of data filtering in either reader 1 or reader 2 . The result proves that HSMURFED method is effective in the application of the proposed system.

\section{Conclusions}

Through the investigation and discussion on the method of RFID data collection and filtration, in order to improve the redundant data filtering, a new method which is based on the adaptive sliding window and Euclidean distance is proposed. Through the comparative analysis of the experimental data, the results show that the method can improve the accuracy of the RFID unreliable data filtering and largely reduce the percentage of false redundancies while maintaining the time-consuming data filtering. In addition, this result proves that HSMURFED method is effective in the application of the proposed system.

Acknowledgement This work is supported by the foundation of Science and Technology Commission of Shanghai Municipality (Grant No. 13521103902).

\section{Reference}

1. Mi ZQ (2011) Radio frequency identification (RFID) technology and application. Publishing House of Electronics Industry, Beijing

2. Xia XF, Xuan LJ (2011) RFID unreliable data cleaning strategy under shunting mechanism. Comp Sci 38(10A):22-25

3. Monge AE. An adaptive and efficient algorithm for detecting approximately duplicate database records. http://citeseer.ni.nec. com/monge00adaptive.html. Accessed 30 Oct 2012

4. Jeffery SR, Franklin MJ, Garofalakis MN (2008) An adaptive RFID middleware for supporting Me tap hysical data independence. Proc of Very Large Datebase, Auckland, New Zealand, pp 256-289

5. Chen HQ, Ku WS, Wang HX (2010) Sun minte leveraging spatio-temporal redundancy for RFID data cleansing. In:
Proceedings of Special Interest Group on Management of Data, Indiana, USA, pp 51-62

6. Carbunar B, Ramanathan M, Koyuturk M et al (2005) Redundant reader elimination in RFID systems. In: The 2nd annual IEEE communications society conference on sensor and Ad Hoc communications and networks, 26-29 Sept 2005, pp 176-184

7. Jeffery SR, Alonso G, Franklin MJ et al (2006) A pipelined framework for online cleaning of sensor data streams. In: Proceedings of the 22nd International Conference on Data Engineering (ICDE), Atlanta, Georgia, USA, p 140

8. Gu Y, Yu G, Zhang TC (2007) RFID complex event processing techniques. Front Comp Sci Technol 1(3):255-267

9. Jeffery SR, Garofalakis M, Franklin M (2006) Adaptive cleaning for RFID data streams. In: Proceedings of the 32nd international conference on very large data bases (VLDB), Seoul, Korea, pp 163-174

10. Ma Y, Zhang YY (2009) Based on sliding window of RFID data stream tabbed cleaning algorithm. Sci Technol Eng 9(5):11651171

11. Jiu LY, Shan GQ (2013) An effective multi-RFID readers redundant data cleaning algorithm. J Northwest Polytech Univ 29(3):6470-6473

12. Chen XH, Wang X (2013) An improved algorithm in redundant data cleaning based on RFID middleware. Microelectron Comp 30(7): 154-158

13. Chen H, Ku WS, Wang HX et al (2010) Leveraging spatiotemporal redundancy for RFID data cleansing. In: Proceedings of the 2010 ACM SIGMOD international conference on management of data SIGMOD, New York, USA, pp 51-62

14. Li X (2013) Research on RFID middleware data cleaning technology. Dissertation, Beijing Jiaotong University

15. Han JW, Kamber M (2011) Data mining concepts and techniques. Morgan Kaufmann Press, Boston

16. Meng LY, Yu FQ (2010) RFID data cleaning based on adaptive window. In: Proceedings of the 2nd International Conference on Future Computer and Communication. Wuhan, China, pp 746-749

17. Eom KH, Lee SJ (2011) Improved Kalman filter method for measurement noise reduction in multi sensor RFID systems. Sensors, pp 10266-10282

18. Ning HS (2012) RFID major projects and national IOT. China Machinery Industry Press, Beijing

19. Chen L (2013) RFID middleware research data cleaning methods. Dissertation, Wuhan University of Technology

20. Shilei LV, Yu ZS (2012) A kind of RFID system based on middleware reader to redundancy algorithm. Electron $\mathrm{J}$ 40(5):965-970 